\title{
New Contribution to Scattering of Weakly Interacting Massive Particles on Nuclei
}

\author{
Gary Prézeau, Andriy Kurylov, Marc Kamionkowski, and Petr Vogel \\ Division of Physics, Mathematics, and Astronomy, California Institute of Technology, Pasadena, California 91125, USA
}

(Received 3 September 2003; published 3 December 2003)

\begin{abstract}
A weakly interacting massive particle (WIMP) is perhaps the most promising candidate for the dark matter in the Galactic halo. The WIMP detection rate in laboratory searches is fixed by the cross section for elastic WIMP-nucleus scattering. Here we calculate the contribution to this cross section from twonucleon currents from pion exchange in the nucleus and show that it may, in some cases, be comparable to the one-nucleon current that has been considered in prior work and perhaps help resolve the discrepancies between the various direct dark-matter search experiments. We provide simple expressions that allow these new contributions to be included in current calculations.
\end{abstract}

DOI: 10.1103/PhysRevLett.91.231301

A neutral, stable, weakly interacting massive particle (WIMP) with mass near the electroweak scale is one of the most natural dark-matter candidates [1,2] because it has a cosmological density comparable to that contributed by halo dark matter. The most widely studied WIMP candidate is the neutralino - a linear combination of the supersymmetric partners of the photon, $Z$, and Higgs bosons - in the minimal supersymmetric extension of the standard model (MSSM). There are, however, other possibilities, including the sneutrino (the neutrino's superpartner), heavy neutrinos, Kaluza-Klein modes in models with universal extra dimensions, etc.

If such WIMPs exist, one way to observe them is directly via detection of the $\mathcal{O}(30 \mathrm{keV})$ recoil energy imparted to a nucleus in a low-background detector when a WIMP collides with the nucleus $[3,4]$. The predicted event rate for these searches depends primarily on the cross section for elastic WIMP-nucleus scattering. Typically, a WIMP-nucleon interaction is derived from the WIMP-quark interaction that appears in the MSSM (or other WIMP-theory) Lagrangian. It has long been known that for supersymmetric models - and recently shown for more general pointlike WIMPs [5] - the WIMP can couple to the nucleon with either a spinindependent (SI) or spin-dependent (SD) interaction. Nuclear physics then allows the WIMP-nucleus cross section to be derived from the SI and SD WIMP-nucleon interactions.

In this work, we use an effective field-theory description of nuclear interactions to consider the contribution to WIMP-nucleus scattering from two-nucleon currents, heuristically, the coupling of WIMPs to the virtual pions that hold the nucleus together. Although this contribution is, as one might expect, small in many cases, for SI interactions it is usually non-negligible and sometimes comparable to or even bigger than the one-nucleon current usually considered.

We begin with the underlying WIMP-quark interaction Lagrangian, construct effective hadronic operators, and organize them according to the order in $q / \Lambda_{\mathrm{H}}$ with $\Lambda_{\mathrm{H}} \sim$ $1 \mathrm{GeV}$, a hadronic scale such as the nucleon mass or the
PACS numbers: 95.35.+d, 13.75.Cs, 25.90.+k

chiral symmetry breaking scale, and $q \sim m_{\pi}$, the pion mass with $q$ being the momentum transfer or the Fermi momentum of the nucleons inside the nucleus. We then focus on the neutralino to perform an explicit evaluation of the two-nucleon scattering amplitude and we estimate the range for its magnitude.

The two-nucleon contributions will have a long-range piece through the exchange of pions and a contact piece as shown in the diagrams of Fig. 1, where the WIMP is denoted by $\chi$. The long-range contributions are enhanced compared to the two-nucleon contact interaction as can be seen from power counting in the small momentum $q$. To show this, we first organize the $\mathcal{X}$-hadron vertices in powers of $q$ using chiral perturbation theory [6] and the fact that the momentum transferred by the WIMP is $\sim 100 \mathrm{MeV}$ or less for WIMP masses $\sim 100 \mathrm{GeV}$ or less. The leading order (LO) quark operators should therefore induce effective WIMP-hadron vertices that do not involve derivatives of the pion fields or pion mass insertions [which are always quadratic and therefore do not contribute at LO or next-to-leading order (NLO)]; the NLO operators would involve a single derivative of the pion field; the next-to-next-to-leading order (NNLO) would involve two derivatives or pion mass insertions; and so on [7]. Thus, counting pion propagators as $\mathcal{O}\left(1 / q^{2}\right)$ and the pion-nucleon vertex as $\mathcal{O}(q)$, we find that the matrix element of Fig. 1(a) $\sim \mathcal{O}\left(K_{\pi \pi} q^{-2}\right)$, Figs. 1(b) and 1(c) $\sim \mathcal{O}\left(K_{N N \pi} q^{-1}\right)$, Fig. $1(\mathrm{~d}) \sim \mathcal{O}\left(K_{N^{4}} q^{0}\right)$, where the $K_{i}$ denote the order of the $\mathcal{X}$-hadron vertices. In general, the LO $\chi$-hadron vertex in each diagram will have $K_{i} \sim$ $\mathcal{O}\left(q^{0}\right)$, though in certain cases symmetry considerations of the underlying particle-physics model require that the LO vertex vanish [7]. Thus, the long-range two-nucleon contributions of Figs. 1(a) and 1(b) and 1(c) are enhanced by $1 / q^{2}$ and $1 / q$, respectively, relative to the short-range operator of Fig. 1(d). In what follows, we at most consider NLO contributions to the scattering amplitude and henceforth neglect the contact term. Therefore, we consider all terms in $K_{\pi \pi}$ and $K_{N N \pi}$ to $\mathcal{O}(q)$ and $\mathcal{O}(1)$, respectively.

We now construct the $\mathcal{X}$-hadron effective Lagrangian from an underlying $\mathcal{X}$-quark effective Lagrangian where 


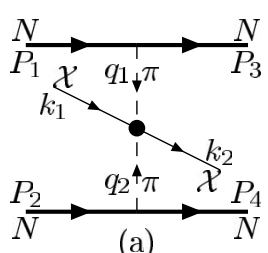

(a)

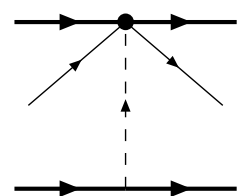

(b)

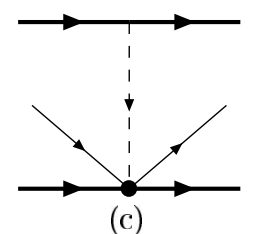

(c)

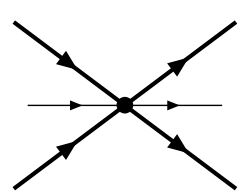

(d)

FIG. 1. Two-nucleon diagrams that contribute to WIMP-nucleus scattering where the WIMP is generally denoted by $\chi$. Graph (a) is of $\mathcal{O}\left(1 / q^{2}\right)$, graphs (b) and (c) are of $\mathcal{O}(1 / q)$, while the contact term of graph (d) is of $\mathcal{O}(1)$. The exchange diagrams are not included. The filled circles represent the nonstandard model vertices.

$\chi$ is a general neutral WIMP field with arbitrary spin, isospin, and parity (note that we are not yet specializing to the neutralino). The most general set of $C P$-conserving interactions between WIMP and quark currents is

$$
\begin{aligned}
\mathcal{L}_{\chi_{q}=G_{\mathrm{F}} \sum_{q}[} & a_{1}^{q} S \bar{q} q+a_{2}^{q} \mathcal{P} \bar{q} \gamma_{5} q \\
& +\mathcal{V}^{\mu} \bar{q} \gamma_{\mu}\left(a_{3}^{q}+a_{4}^{q} \gamma_{5}\right) q \\
& +\mathcal{A}^{\mu} \bar{q} \gamma_{\mu}\left(a_{5}^{q}+a_{6}^{q} \gamma_{5}\right) q \\
& \left.+a_{7}^{q} \mathcal{T}^{\mu \nu} \bar{q} \sigma_{\mu \nu} q\right],
\end{aligned}
$$

where the $S, \mathcal{P}, \mathcal{V}^{\mu}, \mathcal{A}^{\mu}$, and $\mathcal{T}^{\mu \nu}$ are, respectively, linear combinations of scalar, pseudoscalar, vector, axialvector, and tensor operators constructed from $\chi$ and arbitrary constants. The constants $a_{i}^{q}$ are parametrized in terms of the Fermi constant $G_{\mathrm{F}}=\sqrt{2} g^{2} /\left(8 M_{\mathrm{W}}^{2}\right)$ (where $M_{\mathrm{W}}$ is the $W$-boson mass) and depend on the particular particle-physics model (such as the MSSM) used to generate the $\mathcal{X}$-quark Lagrangian. The inclusion of $n$-body quark operators with $n>1$ would induce further suppression in a heavy scale such as $M_{\mathrm{W}}$ and are excluded. In the following, we neglect the $a_{2}^{q}$ term which is generally suppressed by an inverse power of the WIMP mass. Also, only $\mathrm{SU}(2)$ isospin symmetry is considered since kaon-exchange currents are suppressed in elastic WIMPnucleus scattering. We therefore truncate the sum in Eq. (1) to up and down quarks when considering vertices that contribute to exchange currents.

The Lagrangian of Eq. (1) generates various $\mathcal{X}$-hadron interaction vertices. For the particular problem of $\chi$-nucleus scattering at NLO, we focus on the following vertices: $\pi^{2} \chi^{2}, \pi N^{2} \chi^{2}$ (which contribute to the twonucleon current at NLO), and $N^{2} \chi^{2}$ (the one-nucleon contribution to the scattering amplitude). The corresponding $\mathcal{X}$-hadron Lagrangian has the general form

$$
\mathcal{L}_{\chi}=\mathcal{L}^{\pi \pi}+\mathcal{L}^{\pi N^{2}}+\mathcal{L}^{N^{2}}
$$

the last of these is that which has been considered in prior work, while the first two are new. The three parts of $\mathcal{L}_{\chi}$ in Eq. (2) are characterized by parameters $b_{i}, c_{j}, d_{k}$, respectively, which in turn depend on $a_{i}^{q}$ of Eq. (1). The precise shape of this general Lagrangian therefore depends on the $\mathcal{X}$-quark Lagrangian and we now proceed to construct each term in Eq. (2) in turn. $\pi^{2} \chi^{2}$ vertex.-Up to NLO in powers of the pion momentum, the $\pi^{2} \chi^{2}$ Lagrangian looks like

$$
\mathcal{L}^{\pi \pi}=b_{\mathrm{S}} \overrightarrow{\boldsymbol{\pi}} \cdot \overrightarrow{\boldsymbol{\pi}} S+b_{\mathrm{v}} i\left(\overrightarrow{\boldsymbol{\pi}} \times \partial^{\mu} \overrightarrow{\boldsymbol{\pi}}\right)_{3} \mathcal{V}_{\mu} .
$$

In Eq. (3), only the terms with two WIMPs are kept in the expansion of $S, \mathcal{P}, \mathcal{V}^{\mu}$, and $\mathcal{A}^{\mu}$. To derive an expression for $b_{\mathrm{s}}$, we use the matrix element,

$$
b_{\mathrm{S}}\left\langle\pi^{a}|\overrightarrow{\boldsymbol{\pi}} \cdot \overrightarrow{\boldsymbol{\pi}}| \pi^{a}\right\rangle=G_{\mathrm{F}}\left\langle\pi^{a}\left|\sum_{q=u, d} a_{1}^{q} \bar{q} q\right| \pi^{a}\right\rangle,
$$

from which we obtain, using soft-pion techniques [8],

$$
b_{\mathrm{s}}=\frac{G_{\mathrm{F}} m_{\pi}^{2}}{2\left(m_{u}+m_{d}\right)}\left(a_{1}^{u}+a_{1}^{d}\right),
$$

where the $m_{i}$ are current-quark masses. The sum of the quark masses is given in Ref. [9] as $5 \mathrm{MeV}<m_{u}+m_{d}<$ $11 \mathrm{MeV}$ and we use the average value of $8 \pm 3 \mathrm{MeV}$.

For the NLO term of Eq. (3), we use the conservation of the vector current (CVC) to write $b_{v}=G_{\mathrm{F}}\left(a_{3}^{u}-a_{3}^{d}\right)$. Note that in the MSSM there is no contribution from $a_{3}^{q}$.

$\pi N^{2} \chi^{2}$ vertex. - The NLO two-nucleon currents receive contributions only from the $a_{45}^{q}$ terms in Eq. (1) (the other possible terms contribute at NNLO) yielding

$$
\begin{aligned}
\mathcal{L}^{\pi N^{2}}= & c_{\mathrm{v}} \bar{N} \gamma^{\mu} i(\vec{\tau} \times \vec{\pi})_{3} N \mathcal{V}_{\mu} \\
& +c_{\mathrm{a}} \bar{N} \gamma^{\mu} \gamma^{5} i(\vec{\tau} \times \vec{\pi})_{3} N \mathcal{A}_{\mu},
\end{aligned}
$$

where $N$ is the nucleon isospinor. To extract the hadronic scales that appear as one matches the quark- $\mathcal{X}$ Lagrangian to the hadron- $\mathcal{X}$ Lagrangian, we can use dimensional analysis and the scaling rule $[7,10]$

$$
\left(\frac{\bar{N} N}{\Lambda_{\mathrm{H}} f_{\pi}^{2}}\right)^{k}\left(\frac{\partial^{\mu}}{\Lambda_{\mathrm{H}}}\right)^{l}\left(\frac{\pi}{f_{\pi}}\right)^{m} \frac{G_{\mathrm{F}}}{\Lambda_{\mathrm{H}}}\left(\Lambda_{\mathrm{H}} f_{\pi}\right)^{2},
$$

where $f_{\pi} \cong 92.4 \mathrm{MeV}$ is the pion decay constant and $(k, l, m)$ refer to the form of the hadronic part. The $c_{i}$ 's of Eq. (6) which have one-nucleon current and one pion [hence, $(k, l, m)=(1,0,1)]$ can be rewritten $c_{\mathrm{v}, \mathrm{a}}=$ $G_{\mathrm{F}}\left(a_{4,5}^{u}-a_{4,5}^{d}\right) \delta_{\mathrm{v}, \mathrm{a}} / f_{\pi}$, where $\delta_{\mathrm{v}}$ is $\mathcal{O}(1)$ and from CVC we have $\delta_{\mathrm{a}}=-g_{\mathrm{A}} / 2$ with $g_{\mathrm{A}}=1.27$, the usual axial pion-nucleon coupling. Note that in the MSSM, $c_{\mathrm{v}}=$ $c_{\mathrm{a}}=0$.

$N^{2} \chi^{2}$ vertex.-The one-nucleon contribution to the scattering amplitude is traditionally the only term considered and the full $N^{2} \chi^{2}$ Lagrangian is given in Ref. [5]. 
In contrast to the two-nucleon case, the sum in Eq. (1) is now over all quark flavors. At LO and ignoring the pionpole term for simplicity, we have

$$
\begin{aligned}
\mathcal{L}^{N^{2}} \cong & S \bar{N}\left(d_{\mathrm{s}}^{0}+d_{\mathrm{s}}^{1} \tau^{3}\right) N+\mathcal{V}_{\mu} \bar{N} \gamma^{\mu}\left(d_{\mathrm{v}}^{0}+d_{\mathrm{v}}^{1} \tau^{3}\right) N \\
& +\mathcal{A}_{\mu} \bar{N} \gamma^{\mu} \gamma^{5}\left(d_{\mathrm{a}}^{0}+d_{\mathrm{a}}^{1} \tau^{3}\right) N \\
& +\mathcal{T}_{\mu \nu} \bar{N} \sigma^{\mu \nu}\left(d_{\mathrm{t}}^{0}+d_{\mathrm{t}}^{1} \tau^{3}\right) N
\end{aligned}
$$

where [with $(k, l, m)=(1,0,0)]$

$$
d_{i}^{0}=\frac{G_{\mathrm{F}}}{2}\left[\left(a_{j}^{u}+a_{j}^{d}\right) \epsilon_{i}^{0}+2 \sum_{s, b, c, t} a_{j}^{q} \epsilon_{i}^{q}\right],
$$

and $d_{\mathrm{i}}^{1}=G_{\mathrm{F}}\left(a_{j}^{u}-a_{j}^{d}\right) \epsilon_{i}^{1} / 2$, with $j=\{1,3,6,7\}$ for $i=$ $\{\mathrm{s}, \mathrm{v}, \mathrm{a}, \mathrm{t}\}$, respectively. The $\epsilon$ 's are $\mathcal{O}(1)$ except for $\epsilon_{\mathrm{s}}^{1}=\langle N|\bar{u} u-\bar{d} d| N\rangle$ - where $N$ can be either a neutron or a proton-which is small numerically and will henceforth be neglected. For the scalar terms, we have in particular [11,12]

$$
\begin{gathered}
\epsilon_{\mathrm{s}}^{0}=\langle N|\bar{u} u+\bar{d} d| N\rangle \cong 16 \pm 8 \\
\epsilon_{\mathrm{s}}^{s}=\langle N|\bar{s} s| N\rangle \approx(0.04-0.2) \epsilon_{\mathrm{s}}^{0} \\
\epsilon_{\mathrm{s}}^{Q}=\langle N|\bar{Q} Q| N\rangle=\frac{2}{27} \frac{M}{M_{Q}}\left(1-\frac{m_{u}+m_{d}}{2 M} \epsilon_{\mathrm{s}}^{0}-\frac{m_{s}}{M} \epsilon_{\mathrm{s}}^{s}\right),
\end{gathered}
$$

where $Q=c, b, t$. Although Ref. [12] suggests the larger value for $\epsilon_{\mathrm{s}}^{s} \sim 0.2 \epsilon_{\mathrm{s}}^{0}$, the error is very substantial and we use the more central value $\epsilon_{\mathrm{s}}^{s} \sim 0.1 \epsilon_{\mathrm{s}}^{0}$.

MSSM.-In the MSSM, the WIMP is the Majorana spin- $1 / 2$ neutralino which couples to quarks according to the low-energy Lagrangian of Eq. (1) with $a_{3}^{q}=a_{4}^{q}=$ $a_{5}^{q}=a_{7}^{q}=0$ to LO [13]. Since we are interested in comparing the one- and two-nucleon scalar-scalar interactions of Eq. (1) we will not consider the axial-axial $a_{6}^{q}$ term and will focus only on $a_{1}^{q}$, which in the MSSM is given by

$$
a_{1}^{q}=2 \sqrt{2} \frac{m_{q}}{M_{\mathrm{W}}} S_{q},
$$

where $m_{q}$ is the quark mass and the $S_{q}$ 's are dimensionless and are usually larger than 10 in the MSSM [13]. The LO SI $\chi^{2}$-hadron Lagrangian generated by the scalar currents is simply

$$
\mathcal{L}_{0}^{\chi \text { had }}=\left(d_{\mathrm{s}}^{0} \bar{N} N+b_{\mathrm{s}} \vec{\pi} \cdot \vec{\pi}\right) \bar{\chi} \chi .
$$

Therefore, in the MSSM, an important modification of the SI $\chi^{2}$-hadron Lagrangian is the new $\pi^{2} \chi^{2}$ term at LO. This term, through the two-nucleon diagram of Fig. 1(a), can give a contribution comparable in size to the onenucleon scattering amplitude.

The calculation of the diagram in Fig. 1(a) is straightforward [7]: we use the Feynman rules to evaluate the amplitude for two particular nucleons inside the nucleus and we take the nonrelativistic limit; we then Fourier transform the result and obtain the operator

$$
M_{0}^{\pi \pi} \simeq i \frac{S_{\pi} m_{\pi}}{3 \sqrt{2} M^{2}} \frac{g_{\pi N N}^{2}}{4 \pi} G_{\mathrm{F}} \bar{\chi} \chi \mathcal{O}_{0}^{\pi \pi}\left(\vec{x}_{1}, \ldots, \vec{x}_{4}\right),
$$

where $M$ is the nucleon mass, $g_{\pi N N}^{2} /(4 \pi) \cong 13.7$ is the strong pion-nucleon coupling constant, and the nuclear operator is given by

$$
\begin{aligned}
\mathcal{O}_{0}^{\pi \pi}\left(\vec{x}_{1}, \ldots, \vec{x}_{4}\right)= & -\delta\left(\vec{x}_{1}-\vec{x}_{3}\right) \delta\left(\vec{x}_{2}-\vec{x}_{4}\right) \times\left(\xi_{3, \alpha}^{\dagger} \xi_{1, \beta}\right)\left(\xi_{4, \phi}^{\dagger} \xi_{2, \delta}\right)\left(\eta_{3, \sigma}^{\dagger} \eta_{1, \epsilon}\right)\left(\eta_{4, \gamma}^{\dagger} \eta_{2, \zeta}\right) \\
& \times \frac{1}{x}\left[\mathrm{~F}_{1}(x) \vec{\sigma}_{\alpha \beta} \cdot \vec{\sigma}_{\phi \delta}+\mathrm{F}_{2}(x) T_{\alpha \phi, \beta \delta}\right] \vec{\tau}_{\sigma \epsilon} \cdot \vec{\tau}_{\gamma \zeta},
\end{aligned}
$$

where the $\xi$ 's and $\eta$ 's are spinors and isospinors, respectively, $T_{\alpha \phi, \beta \delta} \equiv 3 \overrightarrow{\boldsymbol{\sigma}}_{\alpha \beta} \cdot \hat{\boldsymbol{\rho}} \overrightarrow{\boldsymbol{\sigma}}_{\phi \delta} \cdot \hat{\boldsymbol{\rho}}-\overrightarrow{\boldsymbol{\sigma}}_{\alpha \beta} \cdot \overrightarrow{\boldsymbol{\sigma}}_{\phi \delta}$ and

$$
\begin{gathered}
\mathrm{F}_{1}(x)=(x-2) e^{-x}, \quad \mathrm{~F}_{2}(x)=(x+1) e^{-x}, \\
S_{\pi}=\frac{m_{\pi}^{2}}{\left(m_{u}+m_{d}\right)}\left(S_{u} \frac{m_{u}}{M_{\mathrm{W}}}+S_{d} \frac{m_{d}}{M_{\mathrm{W}}}\right),
\end{gathered}
$$

where $x=m_{\pi} \rho$ is proportional to the distance between the two nucleons with $\vec{\rho}=\vec{x}_{1}-\vec{x}_{2}, \rho=|\vec{\rho}|$, and $\hat{\rho}=$ $\vec{\rho} / \rho$. In Eq. (16), $\vec{\sigma}$ and $\vec{\tau}$ are spin and isospin Pauli matrices, respectively.

The contribution to the WIMP-nucleus scattering will therefore be

$$
\mathcal{A}^{\pi \pi}=i \frac{S_{\pi} m_{\pi}}{3 \sqrt{2} M^{2}} \frac{g_{\pi N N}^{2}}{4 \pi} G_{\mathrm{F}} \mathcal{N}_{0}^{\pi \pi},
$$

and $\mathcal{N}_{0}^{\pi \pi}=\left\langle Z, A\left|\sum \mathcal{O}_{0}^{\pi \pi}\right| Z, A\right\rangle / 2$, where the sum is over all nucleon pairs inside the nucleus, $A$ is the number of nucleons, and $Z$ is the charge of the nucleus.
On the other hand, the one-nucleon contribution will simply be $\mathcal{A}^{N N}=2 i d_{\mathrm{s}}^{0} A$. Taking the ratio of the twonucleon to one-nucleon contribution, we obtain

$$
\begin{gathered}
\frac{\mathcal{A}^{\pi \pi}}{\mathcal{A}^{N N}} \cong(0.21 \pm 0.08) r \frac{\mathcal{N}_{0}^{\pi \pi}}{A}, \quad r \equiv \frac{S_{u} m_{u}+S_{d} m_{d}}{S^{N N}}, \\
S^{N N} \equiv \frac{1}{2}\left(S_{u} m_{u}+S_{d} m_{d}\right) \epsilon_{\mathrm{s}}^{0}+\sum_{s, c, b, t} S_{q} m_{q} \epsilon_{\mathrm{s}}^{q} .
\end{gathered}
$$

Note that we did not include contributions from heavy quarks with extra, inverse powers of the squark masses for simplicity [1] and that the error in the overall factor of 0.21 comes from the uncertainty of the current-quark masses. Looking at the special case where all the $S_{q}$ are equal and cancel in the ratio $r$ with $m_{s}=115 \pm 55 \mathrm{MeV}$, we find $0.002<(0.21 \pm 0.08) r<0.006$. Since there exist regions of the MSSM parameter space where the $S_{q}$ 's have 
opposite signs, the ratio $r$ can be greater than one if cancellations occur. For example, with $S_{u}=S_{d}=S_{s}=$ $-S_{c}=-S_{b}=-S_{t}, m_{s} \epsilon_{\mathrm{s}}^{s}=115 \mathrm{MeV}$, and $\left(m_{u}+m_{d}\right) /$ $2 \epsilon_{\mathrm{s}}^{0}=60 \mathrm{MeV}, r \cong 1.5$. Alternatively, if all the $S_{q}$ 's have the same sign, a cancellation could still occur with the extra heavy quark terms not included in Eq. (21) if they are of similar magnitudes but opposite in sign.

We estimated $\mathcal{N}_{0}^{\pi \pi}$ in the independent particle approximation assuming closed shells and $N=Z$, where $N$ is the number of neutrons. We performed the calculation in two different mean-field potentials: a squarewell and a harmonic-oscillator potential. To account for the hard core, we used Jastrow functions \{defined [ $1-$ $\left.\exp \left(-a \rho^{2}\right)\left(1-b \rho^{2}\right)\right]$ with $a=1.1 \mathrm{fm}^{-2}$ and $b=$ $\left.0.68 \mathrm{fm}^{-2}\right\}$ as well as a simple integral lower cutoff at $0.5 \mathrm{fm}$. We found that the matrix element is approximately linear in $A$ for nucleons in a harmonic potential and exactly linear in $A$ for nucleons trapped in a square well. We also found a minimum value of $\mathcal{N}_{0}^{\pi \pi} \cong A$ in the harmonic-oscillator potential with Jastrow functions and a maximum value of $\mathcal{N}_{0}^{\pi \pi} \cong 2 A$ for the square-well potential with the lower cutoff at $0.5 \mathrm{fm}$. We estimate that $\mathcal{N}_{0}^{\pi \pi} / A \approx 1.5 \pm 0.5$ although the error can be significantly larger due to the fact that the nuclei used in direct dark-matter searches are not closed-shell nuclei and that they have a neutron excess. We also do not take into account spin-orbit coupling. Furthermore, $\mathcal{N}_{0}^{\pi \pi} / A$ could vary substantially from one nucleus to the next. Keeping these issues in mind, we conclude that $\mathcal{N}_{0}^{\pi \pi}$ and $A$ are of the same order and that the ratio in Eq. (20) can be of the order of 1 or larger depending on the values of the MSSM parameters.

In supersymmetric models, the one-nucleon current generically produces roughly equal SI couplings to the proton and neutron [5], which results in a SI amplitude that is proportional to the atomic number of the nucleus. Inclusion of the two-nucleon contributions could change this picture since such contributions might cancel against the one-nucleon contributions. If the ratio of the twonucleon matrix element to the atomic number varies from one nucleus to the next, so will the degree of the cancellation. Thus, when the two-nucleon contribution is taken into account, a dark-matter candidate that appears in the DAMA collaboration but not in other searches [14] is conceivable for a WIMP with SI interactions even within the framework of the MSSM (Ref. [15] provides arguments against a WIMP with SD interactions as an explanation for the DAMA signal).

With $1<\mathcal{N}_{0}^{\pi \pi} / A<2$ and $r \approx 1$, the exchangecurrent contribution is in the range of $10 \%-60 \%$. This is atypical since such currents usually contribute about 5\%-10\% to the amplitude as in $p+n \rightarrow d+\gamma$ [16] and $\nu_{l}+d \rightarrow \nu+p+n$ [17]. Part of the reason the exchange-current contribution is normally small at low energies stems from the fact that the "probe" $-\pi^{2}$ vertex (where the probe can be a photon or a neutrino, for example) is typically suppressed by at least a factor of $q / M \sim 0.1$. In WIMP-nucleus scattering, the situation is different since the $\pi^{2} \chi^{2}$ vertex need not be suppressed by this factor as we showed in Eq. (14). Thus, instead of a $5 \%-10 \%$ effect on the WIMP-nucleus scattering amplitude, the one-nucleon and two-nucleon contributions could be comparable.

In conclusion, we have shown that the two-nucleon and one-nucleon currents can give comparable contributions to the WIMP-nucleus scattering and point out that this result can help resolve the conflict between the various direct dark-matter search experiments.

This work was supported in part by NASA NAG5-9821 and DoE DE-FG03-92-ER40701 and DE-FG0302ER41215.

[1] G. Jungman, M. Kamionkowski, and K. Griest, Phys. Rep. 267, 195 (1996).

[2] L. Bergstrom, Rep. Prog. Phys. 63, 793 (2000).

[3] M.W. Goodman and E. Witten, Phys. Rev. D 31, 3059 (1985).

[4] I. Wasserman, Phys. Rev. D 33, 2071 (1986); A. Drukier, K. Freese, and D. N. Spergel, Phys. Rev. D 33, 3495 (1986); K. Griest, Phys. Rev. D 38, 2357 (1988); P. F. Smith and J. D. Lewin, Phys. Rep. 187, 203 (1990); M. Drees and M. Nojiri, Phys. Rev. D 48, 3483 (1993).

[5] A. Kurylov and M. Kamionkowski, hep-ph/0307185.

[6] J. Gasser and H. Leutwyler, Ann. Phys. (N.Y.) 158, 142 (1984); Nucl. Phys. B250, 465 (1985).

[7] G. Prézeau, M. Ramsey-Musolf, and P. Vogel, Phys. Rev. D 68, 034016 (2003).

[8] J. F. Donoghue, E. Golowich, and B. R. Holstein, Cambridge Monogr. Part. Phys., Nucl. Phys., Cosmol. 2, 1 (1992).

[9] Particle Data Group, K. Hagiwara et al., Phys. Rev. D 66, 010001 (2002).

[10] A. Manohar and H. Georgi, Nucl. Phys. B234, 189 (1984).

[11] P. Schweitzer, hep-ph/0307336.

[12] M. M. Pavan, I. I. Strakovsky, R. L. Workman, and R. A. Arndt, PiN Newslett. 16, 110 (2002).

[13] J. Engel, S. Pittel, and P. Vogel, Int. J. Mod. Phys. E 1, 1 (1992).

[14] DAMA Collaboration, R. Bernabei et al., Phys. Lett. B 480, 23 (2000); R. Bernabei et al., astro-ph/0305542; CDMS Collaboration, R. Abusaidi et al., Phys. Rev. Lett. 84, 5699 (2000); EDELWEISS Collaboration, A. Benoit et al., Phys. Lett. B 545, 43 (2002); N. J. T. Smith, Proceedings of the 4th International Workshop on Identification of Dark Matter (to be published); N. J. C. Spooner, Proceedings of the 3rd Workshop on Neutrino Oscillations and Their Origin (NOON2001) (to be published); DAMA Collaboration, R. Bernabei et al., Phys. Lett. B 436, 379 (1998).

[15] P. Ullio, M. Kamionkowski, and P. Vogel, J. High Energy Phys. 07 (2001) 044.

[16] D. O. Riska, Phys. Rep. 181, 207 (1989).

[17] M. Butler and J.W. Chen, Nucl. Phys. A675, 575 (2000); S. Ando, Y. H. Song, T. S. Park, H.W. Fearing, and K. Kubodera, Phys. Lett. B 555, 49 (2003). 